\title{
A BIOSSEGURANÇA E SEGURANÇA DO PACIENTE NA VISÃO DE ACADÊMICOS DE ENFERMAGEM
}

Telma Elisa CARARRO ${ }^{a}$, Francine Lima GELBCKE ${ }^{\mathrm{b}}$, Luciara Fabiane SEBOLD ${ }^{c}$, Silvana Silveira KEMPFER ${ }^{\mathrm{d}}$, Maria Christina ZAPELINI ${ }^{\mathrm{e}}$, Roberta WATERKEMPER ${ }^{\mathrm{f}}$

\section{RESUMO}

Este estudo objetiva identificar o conhecimento dos acadêmicos de enfermagem frente à temática de segurança do paciente e as relações deste com o ensino da biossegurança. Estudo qualitativo, exploratório, realizado na Universidade Federal de Santa Catarina, com 17 acadêmicos da terceira fase do Curso de Graduação em Enfermagem. Três categorias emergiram após leitura exaustiva das respostas: o cuidado de si e o cuidado do outro; biossegurança e o cuidado com o meio ambiente; biossegurança: educação em saúde e educação permanente no serviço de saúde. Identificou-se a preocupação dos acadêmicos na prevenção de riscos e a relação da biossegurança com o meio ambiente. Apontam o cuidado e o autocuidado como prerrogativas para a segurança do paciente e a educação como proposta para minimizar os riscos. Consideram importante o estudo da biossegurança na graduação, minimizando danos e erros nas condutas.

Descritores: Segurança. Exposição a agentes biológicos. Estudantes de enfermagem. Educação superior.

\section{RESUMEN}

Estudio cualitativo exploratorio, realizado en la Universidad Federal de Santa Catarina, con 17 académicos de la tercera fase del Curso de Grado en Enfermería. Objetiva identificar los conocimientos de los académicos frente a la temática de seguridad del paciente y las relaciones con la enseñanza de la bioseguridad. Surgieron tres categorías: el cuidado de sí mismo y el cuidado del otro; bioseguridad y el cuidado con el medio ambiente; bioseguridad: educación en salud y educación permanente en el servicio de salud. Se ha identificado la preocupación de los académicos en la prevención de riesgos y ellos relacionan la bioseguridad con el medio ambiente; apuntan el cuidado y el autocuidado como prerrogativas para la seguridad del paciente y a la educación como propuesta para minimizar los riesgos. Los académicos consideran importante el estudio de la bioseguridad en el grado, minimizando daños y errores en las conductas.

Descriptores: Seguridad. Exposición a agentes biológicos. Estudiantes de enfermería. Educación superior. Título: La bioseguridad y seguridad del paciente bajo la visión de académicos de enfermería.

\section{ABSTRACT}

This study is aimed at identifying the knowledge of nursing students about the subject area of patient safety and its relationship with the teaching of biosecurity. Exploratory qualitative study conducted at the Universidade Federal de Santa Catarina (Federal University of Santa Catarina), with 17 students in the third phase of the Undergraduate Nursing Program. Three categories emerged after an exhaustive reading of the responses: caring for self and others; biosecurity and care for the environment; biosecurity: health education and continuing education in health services. The following issues were identified: students' concern about risk prevention; care and self care as prerogatives for patient's safety; and education as a proposal for the minimization of risks. The study of biosecurity is considered important in undergraduate studies, minimizing losses and mistakes in the undergraduate students' conduct.

Descriptors: Safety. Exposure to biological agents. Nursing students. Higher, education.

Title: Nursing students' point of view on biosecurity and patient safety.

\footnotetext{
a Telma Elisa Carraro, Doutora, Docente do Programa de Pós-Graduação da Universidade Federal de Santa Catarina - UFSC, Florianópolis, Santa Catarina, Brasil.

b Francine Lima Gelbcke, Doutora, Docente do Programa de Pós-Graduação da UFSC, Florianópolis, Santa Catarina, Brasil.

c Luciara Fabiane Sebold, Doutora, Pós-doutoranda pelo Programa de Pós-Graduação da UFSC, Florianópolis, Santa Catarina, Brasil

d Silvana Silveira Kempfer, Doutoranda pelo Programa de Pós-Graduação da UFSC, Florianópolis, Santa Catarina, Brasil.

e Maria Cristina Zapelini, Mestranda pelo Programa de Pós-Graduação da UFSC, Florianópolis, Santa Catarina, Brasil.

f Roberta Waterkemper, Doutora, Pós-doutoranda pelo Programa de Pós-Graduação da UFSC, Florianópolis, Santa Catarina, Brasil.
} 


\section{INTRODUÇÃO}

A área de conhecimento de biossegurança é relativamente nova, constituindo-se num desafio para os profissionais, especialmente da área da saúde, tendo em vista que as normas de biossegurança são aceitas teoricamente, mas há uma lacuna entre o campo teórico e a prática cotidiana. Neste sentido, é de suma importância que os profissionais de enfermagem adotem as medidas de biossegurança, e para tanto o conhecimento e a conscientização de sua relevância para a segurança e saúde do trabalhador fazem-se necessários, conhecimentos estes que repercutirão também no cuidado ao paciente ${ }^{(1)}$.

Biossegurança é definida como um conjunto de ações destinadas a prevenir, controlar, reduzir ou eliminar riscos inerentes às atividades que possam comprometer a saúde humana, animal e o meio ambiente ${ }^{(2)}$. Este cenário nos remete a Lei $\mathrm{N}^{\circ}$ 11.105 de 25 de março de 2005 que dispõe sobre a Política Nacional de Biossegurança, revogando a Lei $\mathrm{N}^{\circ} 8.974$, de 5 de janeiro de 1995 , e cria a Comissão Técnica Nacional de Biossegurança-CTNBio ${ }^{(2)}$.

$\mathrm{Na}$ área da saúde, considerando-se os riscos ocupacionais a que estão expostos os trabalhadores, a adoção de medidas de biossegurança são fundamentais. No entanto, há uma lacuna na formação dos profissionais de enfermagem acerca desta temática, haja vista que a educação em biossegurança não foi inserida nas disposições legais de formação destes profissionais ${ }^{(1)}$. Este tema tem sido mencionado no curso de Graduação em Enfermagem da Universidade Federal de Santa Catarina, principalmente por considerar-se sua articulação com outro tema extremamente importante - a segurança do paciente, o qual tem sido discutido nacional e internacionalmente em todos os âmbitos da saúde. Esta discussão contribui não só como via para garantir o melhor cuidado, mas também no sentido de assegurar respaldo aos profissionais da saúde, para que suas ações não comprometam a segurança do paciente.

Há que se considerar que o cuidado reduz a incidência de doença e, o dano à saúde encurta o tempo de tratamento e/ou hospitalização, melhora ou mantém a saúde do paciente. Portanto, é de extrema importância se realizar cuidados seguros e neste contexto o enfermeiro é responsável por avaliar o paciente e os riscos ambientais que ameaçam à sua segurança, bem como planejar e intervir apropriadamente para manter um espaço seguro ${ }^{(3)}$.
Frente a este panorama, a Organização Mundial de Saúde (OMS) buscou agregar diversas ações as quais pudessem identificar desajustes nos sistemas de saúde e propor soluções voltadas para a preservação da segurança do paciente, implementando um grande projeto intitulado "Aliança Mundial para a Segurança do Paciente". Este projeto teve como objetivos coordenar, difundir e acelerar a melhoria da segurança do paciente por todo o mundo(t).

No Brasil, em 2007, a Agência Nacional de Vigilância Sanitária (ANVISA) apoiada no modelo do projeto da OMS traçou alguns objetivos com o intuito de identificar os problemas e especificidades da segurança nos serviços de saúde. Autoridades desta organização perceberam a necessidade da participação do Brasil, não apenas pelo grande número de serviços de saúde do país, mas pelas ações prioritárias colocadas em prática, que têm gerado melhorias na assistência à população $0^{(5)}$.

As propostas da ANVISA para segurança do paciente visam à melhoria da qualidade no atendimento hospitalar e a adesão do Brasil à Aliança Mundial para a Segurança do Paciente. A Agência Nacional de Vigilância Sanitária ${ }^{(5)}$ estabeleceu que a segurança do paciente é um dos critérios básicos para alcançar a qualidade de atenção ao paciente, sendo que as diferentes visões dos profissionais ajudam a definir quais são as prioridades nacionais e desta forma construiu-se um projeto adaptado à realidade brasileira nas linhas do programa da $\mathrm{OMS}^{(5)}$. Alguns temas são destacados como a higienização das mãos, a segurança do sangue e hemoderivados em transfusões, a administração segura de medicamentos em procedimentos cirúrgicos, o saneamento e o gerenciamento dos resíduos ${ }^{(5)}$.

É necessário que os educadores busquem incentivar e sensibilizar os profissionais da saúde e principalmente os futuros profissionais para a questão das medidas de biossegurança. As discussões e avanços trazidos pelo estudo da biossegurança são relativamente recentes se considerarmos aspectos históricos da saúde, tendo em vista que impõe desafios não somente à equipe de saúde, mas também na formação de profissionais da saúde, em especial os enfermeiros que atuam diretamente no cuidado às pessoas.

Estas temáticas em questão são abordadas, na teoria e na prática, na terceira fase do curso de Graduação em Enfermagem da Universidade Federal de Santa Catarina, por meio de metodologias ativas, as quais buscam que o aluno articule teoria e prática. 
Para tanto, com o objetivo de avaliar o processo de apreensão do conhecimento, relacionado à temática de biossegurança e segurança do paciente, este estudo teve como questão norteadora: como os acadêmicos de enfermagem compreendem o conteúdo de segurança do paciente e a afinidade deste com o ensino da biossegurança? A partir desta questão definiu-se como objetivo: identificar a conhecimento dos acadêmicos de enfermagem frente à temática de segurança do paciente e as relações deste com o ensino da biossegurança.

\section{METODOLOGIA}

Trata-se de um estudo qualitativo exploratório, realizado em uma universidade pública do sul do Brasil, com 17 acadêmicos do Curso de Graduação em Enfermagem da terceira fase do mesmo, os quais representam metade da turma que estava cursando a disciplina de Fundamentos para o Cuidado Profissional. Os acadêmicos que aceitaram participar do estudo assinaram o Termo de Consentimento Livre Esclarecido.

Os dados foram coletados em sala de aula com este grupo de alunos, após terem sido solicitados a realizarem leituras prévias, não determinadas, acerca das temáticas - segurança do paciente e biossegurança. A partir de então, os acadêmicos responderam de forma livre e aberta a seguinte questão: Qual a importância do estudo da segurança do paciente e as relações com a biossegurança para o enfermeiro no cotidiano hospitalar? Após a coleta dos dados, os mesmos foram organizados em unidades temáticas, categorizados e analisados ${ }^{(6)}$.

Vale ressaltar que este estudo faz parte do contexto da pesquisa intitulada: Metodologias Ativas de Ensino na Formação Profissional em Enfermagem: Repensando as Estratégias para o Ensino - Aprendizagem na Graduação, o qual foi aprovado pelo Comitê de Ética da instituição, sob o protocolo ${ }^{\circ}$ 193/09. Intera-se que foram observados os preceitos éticos relativos à portaria 196/96 que se refere à pesquisa com seres humanos e, para preservar o anonimato dos participantes, os mesmos foram identificados por números.

\section{RESULTADOS}

Após leitura das respostas obtidas, as mesmas foram agrupadas por similaridade em três categorias, a saber: o cuidado de si e o cuidado do outro, englobando as sub-categorias: a importância do uso de Equipamentos de Proteção Individual (EPI), e, biossegurança como forma de evitar acidentes; biossegurança e o cuidado com o meio ambiente; biossegurança: educação em saúde e educação permanente no serviço de saúde.

Assim, na categoria: o cuidado de si e o cuidado do outro, os acadêmicos relatam ser de suma importância o estudo da biossegurança, tanto no que se refere à segurança do trabalhador como a segurança do paciente, destacando ser primordial a abordagem destas questões na graduação. Entendem ser uma forma de proteção para o profissional e para a prevenção de contaminações aos pacientes e familiares, ou seja, uma maneira de preservar a segurança dos mesmos.

É importante para sua proteção e a proteção de seus pacientes para que nenhum dos dois contamine-se.(3)

A biossegurança é importante para manter a integridade física do enfermeiro, para que esse não tenha lesões ou contaminações que coloquem em risco sua saúde. É necessário que o enfermeiro tenha noção e instrução teórica sobre a biossegurança para poder aplicá-la na prática, e que esses profissionais estejam conscientes de como a biossegurança é importante e que a utilizem sempre, dentro de seu ambiente de trabalho.(5)

É importante para proteger os pacientes de contaminação e também para a nossa própria proteção.(2)

\section{A biossegurança é fundamental na nossa profissão, pois estamos em um lugar onde lidasse com bactérias, vírus entre outros microorganismos, e muitas vezes acabamos sendo veículos de transmissão cruzada, tanto no âmbito hospitalar como familiar.(1)}

Identificam a importância do uso dos equipamentos de proteção individual, assim como o destino de materiais contaminados, ações relevantes de prevenção às contaminações tanto dos profissionais como dos pacientes:

As medidas de biossegurança são importantes para o enfermeiro, pois o cuidador deve estar bem para cuidar do paciente; quanto mais o enfermeiro se cuidar, melhor será o cuidado prestado. Medidas como a lavagem das mãos, uso de luvas, jaleco, descarte adequado de pérfuro-cortantes são essenciais para o controle de infecções, tanto para o enfermeiro quanto para o paciente.(12)

Em seu trabalho o enfermeiro é exposto a muitos riscos e efetua muitas práticas que podem prejudicar seus 
pacientes, a sociedade e o meio ambiente em geral. Para que isso não ocorra, é necessário que ele tenha bem claro todas as regras de proteção individual (uso de EPIs, uso de técnicas corretas, assepsia) para não se prejudicar e seus clientes. Também precisam ser observados os cuidados relativos ao descarte de materiais contaminados e uso e manejo corretos de medicamentos que podem causar resistência a microorganismos.(9)

Relacionam a biossegurança como forma de assegurar nas ações de cuidado de enfermagem a segurança do paciente em ambiente hospitalar.

É de suma importância para a área da saúde, pois envolve a prevenção de doenças e transmissão de microorganismos, $e$ através da observação da biossegurança a enfermeira cuida com maior eficiência, e também pode ser vista como uma proteção para si mesma como para o paciente.(13)

A atenção as ações de biossegurança pode ser uma das formas de evitar acidentes de trabalho nas mais diversas esferas do cuidado.

Um dos papéis da enfermagem é a prevenção, e acredito que a biossegurança é muito relacionada com a prevenção pelo fato de que se protegermos a nós mesmos estamos protegendo o paciente e conseqüentemente evitando acidentes de trabalho.(9)

As medidas de biossegurança são de extrema importância para oferecer um ambiente seguro para as pessoas que circulam nele, assim evitando acidentes com pérfuro-cortantes.(17)

Ampliam a importância da biossegurança para além do cuidado com o próprio profissional e com o paciente, indo para a perspectiva ecológica, ao perceberem que a biossegurança e o cuidado com o meio ambiente se entrelaçam no dia a dia.

O cuidado com o meio ambiente também é incluído quando falamos de biossegurança, cuidando para a contaminação hospitalar e do ambiente.(11)

A necessidade de conhecermos a respeito da biossegurança é tanto para a prevenção do paciente quanto do profissional, entretanto devemos nos preocupar e dar atenção ao meio ambiente $e$ as outras pessoas que não estão diretamente envolvidas.(2)

O enfermeiro como membro da equipe de saúde pode orientar outros integrantes, ou até mesmo pessoas que trabalham ou transitam no ambiente hospitalar, e através desta ação evitar danos aos pacientes nas ocorrências de erros que comprometem a segurança do paciente. Neste sentido identificam a biossegurança como educação em saúde e educação permanente.

Proteção do enfermeiro e para que este passe as informações para toda a equipe de saúde, da limpeza, pacientes e acompanhantes, já que este assunto é discutido no curso de enfermagem.(15)

Muitos profissionais não são preparados para as proteções necessárias que devem ser tomadas, e o enfermeiro tem o papel de orientá-los. Além disso, éfundamental ele ter total conhecimento sobre todos os cuidados a serem tomados para um seguro atendimento ao paciente, a fim de evitar contaminação e outros problemas futuros.(12)

\section{DISCUSSÃO}

Apesar do estudo da segurança do paciente ser considerado recente, observa-se que as questões relacionadas com a biossegurança estão interligadas quando se dimensionam os cuidados com a proteção individual e os riscos de contaminação que o paciente e família estão expostos. E neste sentido o aprofundamento do estudo da biossegurança precisa se tornar mais visível aos profissionais da saúde com vistas à segurança do paciente e também dos trabalhadores. O profissional da saúde pode ser considerado como sujeito da aprendizagem e com uma postura crítica-reflexiva do seu ambiente ocupacional. Sendo imprescindível para o ensino de biossegurança o desenvolvimento de habilidades tanto voltadas para o saber fazer, bem como para o saber viver, contemplando as questões éticas ${ }^{(7)}$.

Os dados demonstraram que os acadêmicos consideram ser interessante o estudo da biossegurança discutida na graduação, entendendo ser forma de proteção para o profissional e para os pacientes e familiares, evitando danos e erros em suas condutas. No ambiente hospitalar, em que muitos trabalhadores atuam, existe muitos riscos ocupacionais, e "a adoção de normas de biossegurança no trabalho em saúde é condição fundamental para a segurança dos trabalhadores, qualquer que seja a área de atuação, pois os riscos estão sempre presentes"(1:570).

Outra compreensão dos acadêmicos envolve a importância de utilizar os materiais de proteção individual, assim como o destino de materiais contaminados, sendo que estas ações de prevenção às contaminações e possíveis acidentes, estão relacionadas às normas de biossegurança. Há que se destacar o papel relevante dos profissionais de enfermagem e, 
em especial dos enfermeiros quando desenvolvem as ações de cuidado, pois estão expostos a muitos riscos biológicos, por isto ações como lavagem das mãos, uso de EPI e o descarte adequado de perfuro-cortantes são essenciais para sua proteção. Estes cuidados se destacam, também, quando se pensa na redução dos riscos de transmissão de microorganismos, principalmente o vírus da hepatite B e HIV, e de acidentes ocupacionais por exposição a material biológico e possíveis infecções, sendo necessária a adoção de medidas preventivas ${ }^{(1-10)}$.

Porém, para que as precauções sejam efetivas na prática assistencial, faz-se necessário a correta adesão dos profissionais a estas medidas durante a realização dos cuidados de Enfermagem, independente do diagnóstico do paciente e, mesmo assim, observa-se atualmente a falta de atenção no planejamento e na execução das atividades, a não observância das normas de biossegurança, quer seja pela ausência ou pelo uso inadequado dos $\mathrm{EPI}^{(8)}$.

Nesse sentido os acadêmicos relacionam a biossegurança como forma de cuidar de si por considerarem o profissional um agente que cuida e, que a não observação dos cuidados consigo mesmo pode resultar no descuidado com o paciente, levando a comprometer a segurança do mesmo. Por isso o profissional deve ser responsável não só por sua própria segurança, mas também pela do seu colega e do ambiente, percebendo-se como parte de uma rede de relacionamento ${ }^{(7)}$. A preocupação com a biossegurança envolve ainda a educação permanente, já que o enfermeiro pode orientar aos demais membros das equipes de enfermagem e de saúde. Esse profissional é responsável, muitas vezes, pela capacitação de pessoas que trabalham ou transitam no ambiente hospitalar, executando desde as ações mais simples até as mais complexas.

Há que se considerar que para seguir as normas de biossegurança, o profissional precisa adotar uma atitude de prevenção, que depende de um processo educativo. "A própria equipe de enfermagem tem dificuldade em aderir às medidas de segurança que busquem a proteção ao risco de exposição, subestimando muitas vezes, o próprio risco" (1:570). Portanto, assumir as medidas de biossegurança envolve a necessidade de conhecimento acerca das mesmas, o que implica a articulação da categoria cuidado de si e do outro com a educação permanente, pois para cuidar de si e do outro, o profissional necessita conhecer os riscos e as ações necessárias para exercer a prevenção.
Igualmente, a educação em saúde se faz presente quando refletimos sobre o fazer do enfermeiro. Há que se considerar que os pacientes e familiares, em diferentes situações, precisam ser orientados e é o enfermeiro que tem papel fundamental neste processo de educação.

Um dos problemas relacionados à segurança está no cuidado à saúde e, se refere às constantes falhas operacionais dos sistemas que desviam as ações de enfermeiros para a correção momentânea de tais dificuldades, impedindo que exerçam a enfermagem que aprenderam e idealizaram para seus pacientes e familiares ${ }^{(9)}$. Portanto, o ensino acerca da biossegurança, articulado ao tema de segurança do paciente, não é apenas atual, mas necessário, visando a capacitação dos alunos como futuros profissionais.

A utilização de estratégias educacionais para sensibilizar e para capacitar profissionais para uso das tecnologias precisa ser desenvolvida de maneira ampliada no intuito de fomentar as práticas de biossegurança visando a melhoria do desempenho do enfermeiro e a promoção da segurança do paciente $^{(10)}$. Reforça-se a necessidade da educação permanente dos profissionais da saúde, contemplando os riscos e prevenções de acidentes ocupacionais, uso de equipamentos de proteção individual e coletiva ${ }^{(11)}$. Através destas medidas os profissionais que já atuam nas instituições poderão estar capacitados frente às medidas de biossegurança evitando assim acidentes e colocando em risco, de forma direta e/ ou indireta, a segurança dos pacientes. Em contrapartida os novos profissionais precisam vir de suas formações, neste caso, a graduação em enfermagem, com as medidas de segurança consolidadas em seus saberes e seus fazeres.

\section{CONCLUSÃO}

Percebe-se que os acadêmicos têm conhecimentos sobre as questões de biossegurança, e as relacionam com a preservação da segurança do paciente e sua família em ambiente hospitalar. Consideram as ações de enfermagem como um importante vínculo entre o profissional enfermeiro, a equipe de saúde, os pacientes, familiares e as comunidades em geral, envolvidas nos cuidados à saúde. Por isto, consideram a biossegurança como um modo complexo de prevenir infecções e a disseminação de microorganismos no meio ambiente. E neste sentido, o aprofundamento desta temática precisa tornar-se mais visível a todos os que trabalham 
com seres humanos, principalmente no ambiente de cuidados à saúde e no processo de formação do profissional enfermeiro. Sendo assim, apresenta-se como um tema de grande relevância a ser discutido, aprofundado e praticado na formação profissional.

A utilização das metodologias ativas de ensino aprendizado permite aos alunos a possibilidade de articular teoria e prática e, de certa forma, mobiliza os conhecimentos sobre biossegurança com temas que foram discutidos ao longo da disciplina. Levando em conta que o cuidado é tema central da disciplina Fundamentos para o Cuidado Profissional, desde o cuidado de si e do outro, o cuidado com o meio ambiente e o próprio processo de trabalho - considera-se o enfermeiro como alguém que cuida e educa.

Espera-se que este estudo desperte novos olhares para a formação do profissional enfermeiro no sentido de buscar subsídios para a discussão das temáticas em questão. Ao considerar que estas são, ao mesmo tempo, antigas como a biossegurança e, atuais como a segurança do paciente, acreditamos que suas articulações são necessárias para o cuidado seguro.

\section{REFERÊNCIAS}

1 Andrade AC, Sanna MC. The teaching of Biosafety in Nursing Undergraduation: a literature review. Rev. Bras. Enferm. 2007; 60 (5): 569-72.

2 Brasil. Lei n. 11.105, de 25 de março de 2005: regulamenta os incisos II, IV e V do $\$ 11^{\circ}$ do art. 225 da Constituição Federal, estabelece normas de segurança e mecanismos de fiscalização de atividades que envolvam organismos geneticamente modificados - OGM e seus derivados, cria o Conselho Nacional de Biossegurança - CNBS, reestrutura a Comissão Técnica Nacional de Biossegurança - CTNBio, dispõe sobre a Política Nacional de Biossegurança PNB, revoga a Lei $n^{\circ} 8.974$, de 5 de janeiro de 1995 , e a Medida Provisória no 2.191-9, de 23 de agosto de 2001, e os arts. $5^{\circ}, 6^{\circ}, 7^{\circ}, 8^{\circ}, 9^{\circ}, 10$ e 16 da Lei no 10.814 ,

\section{Endereço da autora / Dirección del autor / Author's address}

Silvana Silveira Kempfer

Rua Jaú Guedes da Fonseca, 292, ap. 101 B, Coqueiros 88080-080, Florianópolis, SC

E-mail: silvanakempfer@yahoo.com.br de 15 de dezembro de 2003, e dá outras providências. Brasília (DF); 2005.

3 Potter PA, Perry AG. Fundamentos de Enfermagem. $7^{\mathrm{a}}$ ed. São Paulo: Editora; 2009.

4 Organización Mundial de la Salud, Asamblea Mundial de la Salud, Comisión B. Assuntos técnicos y sanitários: seguridad del paciente. In: $59^{\mathrm{a}}$ Asamblea Mundial de la Salud: actas resumidas e informes de las comisiones; 2006 mayo 22-27; Ginebra, Suiza. Ginebra: OMS; 2006. p.261-266.

5 Ministério da Saúde (BR). Agência Nacional de Vigilância Sanitária. Anvisa promove debate sobre segurança do paciente no Brasil [Internet]. Brasília (DF); 2008 [citado 2008 maio 15] Disponível em: http://www.anvisa.gov.br/DIVULGA/noticias/2007/171007.htm.

6 Bardin L. Análise de conteúdo. Lisboa: Edições 70; 2008.

7 Pereira MEC, Costa MAF, Borba CM, Jurberg C. Knowledge building in biosafety: a review of the Brazilian academic production on health themes (1989-2009). Saúde Soc.2010; 19(2): 440-448.

8 Alves SSM, Passos JP, Tocantins FR. Accidents with perforate-cutting instruments and nursing workers: a question of biological security. Rev Enferm UERJ. 2009; 17(3):373-7.

9 Pedreira MLG. Nursing interventions and outcomes to ensure patient's safety [Editorial] Acta Paul Enferm. 2009; 22(4). v-vi.

10 Avelar AFM, Peterlini MAS, Onofre PSC, Pettengill MAM, Pedreira MLG. Training of nurses in the use of intravascular ultrasound in peripheral puncture. Acta Paul. Enferm. 2010; 23(3): 433-436.

11 Silva TR, Rocha SA, Ayres JA, Juliani CMCM. Accidents with cutting and piercing materials among nursing professionals at a university hospital. Rev Gaúcha Enferm. 2010; 31(4): 615-622.

Recebido em: 07.11.2011

Aprovado em: 31.08 .2012 